\title{
ON THE NEUMANN PROBLEM \\ FOR SOME SEMILINEAR ELLIPTIC EQUATIONS AND SYSTEMS OF ACTIVATOR-INHIBITOR TYPE
}

\author{
WEI-MING NI AND IZUMI TAKAGI
}

\begin{abstract}
We derive a priori estimates for positive solutions of the Neumann problem for some semilinear elliptic systems (i.e., activator-inhibitor systems in biological pattern formation theory), as well as for semilinear single equations related to such systems. By making use of these a priori estimates, we show that under certain assumptions, there is no positive nonconstant solutions for single equations or for activator-inhibitor systems when the diffusion coefficient (of the activator, in the case of systems) is sufficiently large; we also study the existence of nonconstant solutions for specific domains.
\end{abstract}

1. Introduction. In this paper we are concerned with the Neumann problem for some semilinear elliptic equations and systems. Let $\Omega$ be a bounded domain in $\mathbf{R}^{N}, N \geq 2$, with smooth boundary $\partial \Omega$, and let $n$ denote the unit outer normal to $\partial \Omega$. A typical system of equations which we consider is

$$
\begin{aligned}
& \left.\begin{array}{l}
d \Delta u-u+\frac{u^{p}}{v^{q}}+\sigma=0 \\
D \Delta v-\nu v+\frac{u^{r}}{v^{s}}=0
\end{array}\right\} \text { in } \Omega \\
& \frac{\partial u}{\partial n}=\frac{\partial v}{\partial n}=0 \quad \text { on } \partial \Omega,
\end{aligned}
$$

where $d, D$, and $\nu$ are positive constants, $\sigma$ is a nonnegative constant,

$$
\Delta=\sum_{j=1}^{N} \frac{\partial^{2}}{\partial x_{j}^{2}}
$$

is the $N$-dimensional Laplace operator, and the exponents $p>1, q>0, r>0$, and $s \geq 0$ satisfy

$$
0<(p-1) / q<r /(s+1) .
$$

Moreover, we are only interested in positive solutions since $u$ and $v$ represent the concentrations of certain substances.

The system (1.1)-(1.3) was proposed by Gierer and Meinhardt [5] as a model of biological pattern formation. Stable nonconstant solutions to (1.1)-(1.3) are interpreted as the spatially inhomogeneous state of cells. Here, by the stability of solutions to elliptic equations, we mean the stability viewed as stationary solutions

Received by the editors October 17, 1985.

1980 Mathematics Subject Classification (1985 Revision). Primary 35J25, 35J55, 35J60.

Research supported in part by NSF Grant \#DMS 8200033A01. 
to the corresponding diffusion equations. For biological aspects of the system (1.1)(1.3) we refer to [5 or 8 ].

We shall also consider single equations including

$$
\begin{gathered}
d \Delta u-u+u^{p}=0 \quad \text { in } \Omega, \\
\frac{\partial u}{\partial n}=0 \text { on } \partial \Omega,
\end{gathered}
$$

where $d$ is a positive constant and $p$ satisfies

$$
1<p<N /(N-2),
$$

with the understanding that $1<p<+\infty$ if $N=2$. This problem may be regarded as a model or reduced one for (1.1)-(1.3). In fact, (1.5)-(1.6) plays an important role when we consider (1.1)-(1.3) for $D$ sufficiently large because, as $D \rightarrow+\infty$, it can be reduced to the shadow system:

$$
\begin{gathered}
d \Delta u-u+\frac{u^{p}}{\xi^{q}}+\sigma=0 \quad \text { in } \Omega, \\
\int_{\Omega} u^{r} d x=\nu \xi^{s+1} \\
\frac{\partial u}{\partial n}=0 \text { on } \partial \Omega .
\end{gathered}
$$

In particular, if $\sigma=0$, then $w(x)=\xi^{-q /(p-1)} u(x)$ satisfies (1.5).

For $N=1$, we can justify the reduction to the shadow system and investigate the structure of the solution set of (1.1)-(1.3) in great detail if $D$ is sufficiently large (see [17]). In the case of $N \geq 2$, however, very little is known both for (1.1)-(1.3) and for (1.5)-(1.6).

The purpose of this paper is to derive a priori estimates for positive solutions to the system (1.1)-(1.3) under further restrictions on the exponents. In fact, we shall obtain the following estimates as a special case of the main results proved in $\S 4$ (Theorems 5 and 6).

TheOREM 1. (a) Let $\sigma>0$. Assume that

$$
p / q \leq r /(s+1) \quad \text { and } \quad r \geq \max (p, N(p-1) / 2) .
$$

Then any solution $(u, v)$ to (1.1)-(1.3) satisfies

$$
\begin{gathered}
\|u\|_{C^{\theta}(\bar{\Omega})} \leq C \max \left(1, d^{-\Lambda_{m}}\right), \\
\|v\|_{C^{\theta}(\bar{\Omega})} \leq C \max \left(1, d^{-\Lambda_{m} r /(s+1)}\right) \max \left(1, D^{-1}\right),
\end{gathered}
$$

where $\Lambda_{m}=\sum_{j=0}^{m} p^{j}$ and $m$ is a nonnegative integer defined by (4.12) with $\alpha=r / p$.

(b) Let $\sigma=0$. Assume that

$$
p / q=r /(s+1) \text { and } r / p>N / 2 .
$$

Then any solution $(u, v)$ to (1.1)-(1.3) satisfies

$$
\|u\|_{C^{\theta}(\bar{\Omega})} \leq C \max \left(1, d^{-1}\right) \text {. }
$$

If $0 \leq s<2 /(N-2)$ holds in addition to (1.14), then

$$
\|v\|_{C^{\theta}(\bar{\Omega})} \leq C \max \left(1, d^{-r /(s+1)}\right) \max \left(1, D^{-1}\right) .
$$


Here $C>0$ and $\theta \in(0,1)$ do not depend on $(u, v), d$ or $D ; 2 /(N-2)$ stands for $+\infty$ if $N=2$.

It is perhaps worthwhile mentioning that in Theorem 1, if one is only interested in getting a priori bounds for $\|v\|_{\infty}$, then in case (b), the extra condition on $s$ is not needed (see §5).

To achieve this purpose, we shall first derive a priori estimates for positive solutions to single equations including (1.5)-(1.6) as functions of $d$, which are of independent interest. By making use of these a priori estimates we are able to show that if $d$ is sufficiently large, then there is no nonconstant positive solution of (1.5)-(1.6) or of (1.1)-(1.3) under the assumptions $\sigma>0$ and (1.11). We also consider the existence of nonconstant solutions for some specific domains for (1.5)-(1.6) and for (1.1)-(1.3) under the same restriction.

We remark that, for $N=1$, an a priori bound for solutions to the system (1.1)(1.3) is derived in [17, Lemma 1.2] under the general condition (1.4); while in the cas $N \geq 2$, a priori estimates were obtained for a special choice of the exponents (i.e., $p=2, q=1, r=2$, and $s=0$ ) when $\sigma$ is positive. Our approach here covers the case $\sigma=0$ under a certain restriction on the exponents.

For related works on the Gierer-Meinhardt system (1.1)-(1.3), we refer to the references in $[\mathbf{1 7}]$; for the time-dependent problem, see Rothe [14].

This paper is organized as follows: $\S \S 2$ and 3 are concerned with single equations including (1.5)-(1.6), while $\S 4$ deals with activator-inhibitor systems (to be specified at the beginning of the section) which include (1.1)-(1.3) satisfying (1.11). In $\S 2$ we derive a priori estimates for single equations (Theorem 2 ). In $\S 3$ we prove the nonexistence of positive nonconstant solutions for sufficiently large $d$ (Theorem 3) and consider the existence of nonconstant solutions for specific domains by employing bifurcation theory (Theorem 4). $\S 4$ is divided into two subsections. In subsection 4.1, a priori estimates for activator-inhibitor systems are derived (for $u$ in Theorem 5 and for $v$ in Theorem 6 ). In subsection 4.2, we give some applications of the a priori estimates. Theorem 7 is concerned with the nonexistence of positive nonconstant solutions; while the existence of nonconstant solutions for specific domains is stated in Theorem 8 . Finally we observe that $v$ is close to a constant if $D$ is sufficiently large (Theorem 9).

ACKNOWLEDGMENT. The authors would like to thank Professors E. Fabes and C. E. Kenig for several useful conversations on the elliptic estimates in Lipschitz domains.

Part of this work was done while the second author was visiting the Institute for Mathematics and its Applications, University of Minnesota, whose hospitality he acknowledges. He is also grateful to Professors T. Kotake and K. Masuda for their encouragement.

2. A priori estimates for single equations. In this section we shall derive a priori estimates for nonnegative solutions to the following Neumann problem:

$$
\begin{gathered}
d \Delta u-u+h(u)=0 \quad \text { in } \Omega \\
\frac{\partial u}{\partial n}=0 \quad \text { on } \partial \Omega .
\end{gathered}
$$


Here $d>0$ is a constant; the function $h:[0,+\infty) \rightarrow[0,+\infty)$ is continuous and satisfies

$$
a_{0} u^{p_{0}} \leq h(u) \leq a_{1} u^{p} \quad \text { for sufficiently large } u
$$

with some positive constants $a_{0}$ and $a_{1}$ independent of $u$, where the exponents $p_{0}$ and $p$ satisfy

$$
1<p_{0}<p<N /(N-2)
$$

with the understanding that $N /(N-2)$ denotes $+\infty$ if $N=2$.

Throughout the section we assume that solutions $u$ to $(2.1)-(2.2)$ are of class $C^{2}(\Omega) \cap C^{1}(\bar{\Omega})$. To state our results we need some notations. Define an integer $k$ by

$$
k=\min \left\{j \in Z \mid p^{j}>2 /[N-(N-2) p]\right\} .
$$

Note that $k \geq 1$ because of (A.2). Put

$$
\Lambda_{k}=\sum_{j=0}^{k} p^{j} .
$$

Then the main result in this section is stated as follows.

THEOREM 2. Let $u$ be a nonnegative solution to (2.1)-(2.2). Then $u$ satisfies

$$
\|u\|_{C^{\theta}(\bar{\Omega})} \leq C \max \left(1, d^{-\Lambda_{k}}\right)
$$

for some $\theta \in(0,1)$ and $C>0$ independent of $u$ and $d$.

Theorem 2 is an immediate consequence of the following proposition since we have the continuous embedding $W^{2, q}(\Omega) \subset C^{\theta}(\bar{\Omega}), 0<\theta \leq 2-N / q$, if $q>N / 2$. (For the Sobolev embedding theorems see, e.g., [9, Chapter 3].)

PROPOSITION 2.1. There exist positive numbers $q^{*}>N / 2$ and $C$ depending only on $\Omega, a_{0}, a_{1}, p_{0}$, and $p$ such that

$$
\|u\|_{W^{2, q^{*}}(\Omega)} \leq C \max \left(1, d^{-\Lambda_{k}}\right)
$$

holds for any solution to (2.1)-(2.2).

In the proof of Proposition 2.1, we use the following elliptic estimates for the linear Neumann problem:

$$
\Delta u-u+g=0 \quad \text { in } \Omega, \quad \frac{\partial u}{\partial n}=0 \quad \text { on } \partial \Omega .
$$

LEMMA 2.2. (a) Let $g \in L^{1}(\Omega)$ and let $u \in W^{1,1}(\Omega)$ be a weak solution of (2.7). Then $u \in W^{1, q}(\Omega)$ for all $q \in[1, N /(N-1))$ and

$$
\|u\|_{W^{1, q}(\Omega)} \leq C\|g\|_{L^{1}(\Omega)}
$$

with $C$ independent of $u$.

(b) Let $g \in L^{r}(\Omega)$ with $1<r<+\infty$ and $u$ be a generalized solution of (2.7). Then $u \in W^{2, r}(\Omega)$ and satisfies

$$
\|u\|_{W^{2, r}(\Omega)} \leq C\|g\|_{L^{r}(\Omega)} .
$$


These are standard facts (see, e.g., [3] for the proof of (a) and [1] for (b)).

In what follows, the letter $C$ denotes various generic positive constants which do not depend on $d$ or $u$.

PROOF OF PROPOSITION 2.1.

Step 1 . First of all, we integrate both sides of (2.1) over $\Omega$ and use (2.2) to obtain

$$
\int_{\Omega} h(u) d x=\int_{\Omega} u d x
$$

Thus from (A.1) it follows that

$$
a_{0} \int_{\Omega} u^{p_{0}} d x \leq \int_{\Omega} u d x+C .
$$

Applying Hölder's inequality to the right-hand side leads to $\int_{\Omega} u^{p_{0}} d x \leq C$, so that

$$
\int_{\Omega} u d x \leq C .
$$

From (2.10) and (2.12) we also have

$$
\int_{\Omega} h(u) d x \leq C
$$

Let us put

$$
g(x)=d^{-1} h(u(x))+\left(1-d^{-1}\right) u(x) .
$$

Then (2.1)-(2.2) is written in the form of (2.7) and hence by Lemma 2.2(a) we have

$$
\|u\|_{W^{1, q}(\Omega)} \leq C \max \left(1, d^{-1}\right)
$$

for each $q \in[1, N /(N-1))$ since

$$
\int_{\Omega}|g| d x \leq d^{-1} \int_{\Omega} h(u) d x+\max \left(1, d^{-1}\right) \int_{\Omega} u d x \leq C \max \left(1, d^{-1}\right)
$$

by (2.10) and (2.13).

Now by the Sobolev embedding theorem, we have the continuous embedding $W^{1, q}(\Omega) \subset L^{r}(\Omega)$ with $r=N q /(N-q)$. Since $q<N /(N-1)$, we see that $1 / r=1 / q-1 / N>(1-1 / N)-1 / N=1-2 / N$, i.e., $1<r<N /(N-2)$. Therefore (2.14) yields that for each $r \in(1, N /(N-2))$

$$
\|u\|_{L^{r}(\Omega)} \leq C \max \left(1, d^{-1}\right) .
$$

Step 2. By assumption on $p$, we can choose $r$ so that $p<r<N /(N-2)$ holds. Put $r_{1}=r / p$. Then by making use of (A.1), we see that

$$
\begin{aligned}
\|g\|_{L^{r_{1}}(\Omega)} & \leq d^{-1}\|h(u)\|_{L^{r_{1}}(\Omega)}+\max \left(1, d^{-1}\right)\|u\|_{L^{r_{1}}(\Omega)} \\
& \leq d^{-1}\left(a_{1}\left\|u^{p}\right\|_{L^{r_{1}}(\Omega)}+C\right)+\max \left(1, d^{-1}\right)\|u\|_{L^{r_{1}}(\Omega)} .
\end{aligned}
$$

Noting that $\|u\|_{L^{r_{1}}(\Omega)} \leq C\|u\|_{L^{r}(\Omega)}$ and $\left\|u^{p}\right\|_{L^{r_{1}}(\Omega)}=\|u\|_{L^{r}(\Omega)}^{p}$, we have

$$
\|g\|_{L^{r_{1}}(\Omega)} \leq C d^{-1}\left(\|u\|_{L^{r}(\Omega)}^{p}+1\right)+C \max \left(1, d^{-1}\right)\|u\|_{L^{r}(\Omega)} .
$$

The right-hand side can be estimated by a function of $d$ in virtue of $(2.15)$, so we may conclude by using Lemma 2.2 (b) that

$$
\|u\|_{W^{2, r_{1}}(\Omega)} \leq C \max \left(1, d^{-p-1}\right) .
$$


If we can find an $r \in(p, N /(N-2))$ such that $p / r-2 / N<0$, we are through. In particular, we have proved the proposition for $N=2$.

Step 3. Assume that $1 / r_{1}-2 / N=p / r-2 / N>0$ for all $r \in(p, N /(N-2))$. Then by the Sobolev embedding theorem, $W^{2, r_{1}}(\Omega) \subset L^{s_{1}}(\Omega)$, where

$$
1 / s_{1}=1 / r_{1}-2 / N \text {. }
$$

From (2.17) it follows that

$$
\|u\|_{L^{s_{1}}(\Omega)} \leq C \max \left(1, d^{-p-1}\right) .
$$

Now, we go back to Step 2 with $r_{1}$ replaced by $r_{2}=s_{1} / p$. As in the derivation of (2.16), we see that

$$
\begin{aligned}
\|g\|_{L^{r_{2}}(\Omega)} & \leq C d^{-1}\left(\|u\|_{L^{s_{1}}(\Omega)}^{p}+1\right)+C \max \left(1, d^{-1}\right)\|u\|_{L^{s_{1}}(\Omega)} \\
& \leq C \max \left(1, d^{-p^{2}-p-1}\right)
\end{aligned}
$$

by making use of (2.19). Thus, in view of Lemma $2.2(\mathrm{~b})$, we have from (2.20) that

$$
\|u\|_{W^{2, r_{2}(\Omega)}} \leq C \max \left(1, d^{-p^{2}-p-1}\right) .
$$

If there is an $r \in(p, N /(N-2))$ such that $1 / r_{2}-2 / N<0$, then we obtain the assertion. If not, we repeat Step 3.

After the $(l-1)$ st iteration of Step 3 , we have a sequence $\left\{s_{j}\right\}_{j=0}^{l}$ such that

$$
\left\{\begin{array}{l}
s_{0}=r \\
1 / s_{j}=p / s_{j-1}-2 / N \text { for } j \geq 1
\end{array}\right.
$$

i.e., $1 / s_{j}=2 /[N(p-1)]+p^{j}(1 / r-2 /[N(p-1)])$, and the estimate

$$
\|u\|_{W^{2, s_{l} / p}(\Omega)} \leq C \max \left(1, d^{-\Lambda_{l}}\right) .
$$

By an elementary computation, we see that $k$ defined by (2.3) is the least number for which there exists an $r \in(p, N /(N-2))$ such that $s_{j}>0$ for $j \leq k-1$ and $s_{k}<0$. Therefore we have completed the proof.

REMARK 2.3. For the important special case $h(u)=u^{p}$, we note that if $p \geq$ $(N+2) /(N-2)$, then a priori estimates of the form (2.5) are still open. For the case $p<(N+2) /(N-2)$, it is possible to prove that for each $d$, there exists a constant $C=C(d)$ such that if $u$ is a positive solution of $(2.1)-(2.2)$, then $\|u\|_{L^{\infty}(\Omega)} \leq C(d)$. However, in order to make use of such a priori estimates (e.g. nonexistence theorems for $d>0$ large, such as Theorem 3 below), it is necessary to obtain a somewhat explicit dependence of $C(d)$ as a function of $d$. This is a bit more subtle and is achieved by an entirely different method (compared to the one used here) by C.-S. Lin, W.-M. Ni, and I. Takagi in an ongoing current joint project. Perhaps we should also mention that in contrast to the nonexistence result-Theorem 3 below-we have also obtained existence results for (2.1)-(2.2) in case $d>0$ sufficiently small and $p<(N+2) /(N-2)$. All these results will be published elsewhere in a joint paper with C.-S. Lin.

REMARK 2.4. It is clear that our method of deriving a priori estimates in this section applied to more general equations of the form

$$
\Delta u+f(x, u)=0 \quad \text { in } \Omega, \quad \frac{\partial u}{\partial n}=0 \quad \text { on } \partial \Omega,
$$

with some appropriate conditions on $f(x, u)$. We should remark that our main interest is in the dependence of a priori estimates on the parameter $d$. 
3. Nonconstant solutions of single equations. In this section we consider the existence problem for (2.1)-(2.2) under the assumption that $h(u)$ satisfies, in addition to (A.1) and (A.2), the conditions

$$
h \in C^{1}([0,+\infty))
$$

(A.4) the equation $u=h(u)$ has precisely two solutions $u=0$ and $u=\gamma$, where $\gamma>0$.

Later on we shall impose a further condition:

$$
h^{\prime}(\gamma)>1
$$

Clearly, $u \equiv 0$ and $u \equiv \gamma$ are solutions to (2.1)-(2.2) for all $d>0$. First we show that (2.1)-(2.2) has no other solutions if $d$ is sufficiently large.

THEOREM 3. Suppose that (A.1)-(A.4) are satisfied. Then there exists a $d^{*}>0$ such that if $d>d^{*}$, then $u \equiv \gamma$ is the only positive solution of (2.1)-(2.2).

PrOOF. Let $u$ be a positive solution to (2.1)-(2.2) and decompose $u$ as $u=$ $u_{0}+\phi$, where

$$
u_{0}=|\Omega|^{-1} \int_{\Omega} u d x \text { and } \int_{\Omega} \phi d x=0,
$$

$|\Omega|$ being the volume of $\Omega$. Then from (2.1) we have

$$
d \Delta \phi-\phi+\left(\int_{0}^{1} h^{\prime}\left(u_{0}+t \phi\right) d t\right) \phi=u_{0}-h\left(u_{0}\right) .
$$

Multiply both sides of (3.2) by $\phi$ and then integrate over $\Omega$. Integration by parts gives

$$
d \int_{\Omega}|\nabla \phi|^{2} d x+\int_{\Omega} \phi^{2} d x=\int_{\Omega} \phi^{2}\left(\int_{0}^{1} h^{\prime}\left(u_{0}+t \phi\right) d t\right) d x
$$

by virtue of (3.1).

Now it follows from Theorem 2 that $0<u_{0}+t \phi(x) \leq \max u(x) \leq C \max \left(1, d^{-\Lambda_{k}}\right)$ for $(x, t) \in \bar{\Omega} \times[0,1]$, so that, by (A.3), $\left|h^{\prime}\left(u_{0}+t \phi(x)\right)\right| \leq C$ for $(x, t) \in \bar{\Omega} \times[0,1]$ uniformly in $d$ sufficiently large, say $d \geq 1$.

On the other hand we have the Poincaré inequality for $\phi \in C^{1}(\bar{\Omega})$ :

$$
\int_{\Omega}|\nabla \phi|^{2} d x \geq c_{0} \int_{\Omega} \phi^{2} d x \text { if } \int_{\Omega} \phi d x=0
$$

where $c_{0}$ is the smallest positive eigenvalue of $-\Delta$ under homogeneous Neumann boundary conditions.

Therefore, from (3.3) we see that

$$
\left(1+c_{0} d\right) \int_{\Omega} \phi^{2} d x \leq C \int_{\Omega} \phi^{2} d x
$$

provided $d \geq 1$. Thus $\phi \equiv 0$, i.e., $u$ is a constant if $d>(C-1) / c_{0}$. In view of (A.4), we may conclude that $u \equiv \gamma$, as desired. Q.E.D. 
Next, we show the existence of nonconstant solutions near $u \equiv \gamma$ by applying bifurcation theory. For this purpose, we introduce a space of functions:

$$
X=\left\{u \in C^{2+\theta}(\Omega) \cap C^{1}(\bar{\Omega}) \mid \frac{\partial u}{\partial n}=0 \text { on } \partial \Omega\right\},
$$

where $0<\theta<1$. Let $\mathbf{R}_{+}$denote the set of all positive real numbers. We interpret the problem as finding a pair $(d, u) \in \mathbf{R}_{+} \times X$ satisfying (2.1). The set $\Gamma=$ $\{(d, \gamma) \mid d>0\}$ is.called the branch of trivial solutions. A point $\left(d_{c}, \gamma\right) \in \Gamma$ is said to be a bifurcation point if every neighborhood of $\left(d_{c}, \gamma\right)$ in $\mathbf{R}_{+} \times X$ has a nontrivial solution $(d, u), u \neq \equiv$.

From now on we assume further that (A.5) is also satisfied. Putting $u=\gamma+w$, $w \in X$, we have an equation for $w$,

$$
d \Delta w+\left(h^{\prime}(\gamma)-1\right) w+\eta(w)=0
$$

where $\eta(w)=h(\gamma+w)-\gamma-h^{\prime}(\gamma) w$ satisfies $\eta(0)=\eta^{\prime}(0)=0$. As is easily seen, a necessary condition for $(d, \gamma)$ to be a bifurcation point is that the linear operator appearing in (3.6) has nontrivial kernel. Let $\left\{l_{j}\right\}_{j=0}^{\infty}$ be the eigenvalues of $-\Delta$ under homogeneous Neumann boundary conditions, and $\left\{l_{j}\right\}_{j=0}^{\infty}$ the corresponding eigenfunctions

$$
\begin{gathered}
-\Delta \phi_{j}=l_{j} \phi_{j} \quad \text { in } \Omega, \quad \frac{\partial \phi_{j}}{\partial n}=0 \quad \text { on } \partial \Omega \\
0=l_{0}<l_{1} \leq l_{2} \leq \cdots \leq l_{j} \leq \cdots \uparrow+\infty
\end{gathered}
$$

Then the operator $d \Delta+\left(h^{\prime}(\gamma)-1\right) I$ has nontrivial kernel if and only if $d=d_{j}$, where we define

$$
d_{j}=\left(h^{\prime}(\gamma)-1\right) / l_{j}, \quad j=1,2,3, \ldots
$$

We see that this condition is also sufficient.

PROPOSITION 3.1. (a) Each $\left(d_{j}, \gamma\right)$ is a bifurcation point.

(b) If $l_{j}, j \geq 1$, is a simple eigenvalue, then the solution set near $\left(d_{j}, \gamma\right)$ consisis of exactly two curves $\Gamma$ and $\left\{\left(d_{j}(\varepsilon), u_{j}(\varepsilon)\right)|| \varepsilon \mid<\varepsilon_{0}\right\}$, where $d_{j}(\varepsilon)=d_{j}+O(\varepsilon)$, $u_{j}(\varepsilon)(x)=\gamma+\varepsilon \phi_{j}(x)+o(\varepsilon)$.

PROOF. Assertion (a) is obtained by applying bifurcation theory for gradient operators (see, e.g., Böhme [2, Satz II.1] or Rabinowitz [13]). In fact, we see that $(\tau, w)=(0,0)$ is a bifurcation point for the equation

$$
\Delta w+l_{j} w+\left(l_{j}-\tau\right)\left(h^{\prime}(\gamma)-1\right)^{-1} \eta(w)=\tau w
$$

under homogeneous Neumann boundary conditions by virtue of Theorem 0.2 of [13] which is stated for nonlinear terms independent of $\tau$; however, the proof remains unchanged for our case (cf. Remark 1.25 of [13]). Note that nontrivial solutions $(\tau, w) \in \mathbf{R} \times W^{1,2}(\Omega)$ to the above equation give nonconstant solutions $\left(\left[h^{\prime}(\gamma)-\right.\right.$ 1] $\left./\left(l_{j}-\tau\right), \gamma+w\right)$ to $(2.1)$ and that such solutions turn out to be smooth because of elliptic regularity theorems. Therefore we obtain (a).

Assertion (b) follows from the standard theorem of bifurcation from simple eigenvalues (e.g., Crandall and Rabinowitz [4, Theorem 2.4]). Q.E.D.

In order to obtain more information on the structure of the solution set, we would like to consider (2.1)-(2.2) in a specific domain. 
A rectangle $R=\left\{\left(x_{1}, \ldots, x_{N}\right) \mid 0<x_{j}<a_{j}, j=1, \ldots, N\right\}$ is called simple if $\left\{a_{j}^{-2}\right\}_{j=1}^{N}$ are rationally independent, i.e., $\sum_{j=1}^{N} n_{j} a_{j}^{-2}=0, n_{j} \in Z$, implies $n_{j}=0$ for all $j=1, \ldots, N$. The eigenvalues of $-\Delta$ in $R$ subject to homogeneous Neumann boundary conditions are given by

$$
l_{M}=\pi^{2}\left(m_{1}^{2} / a_{1}^{2}+\cdots+m_{N}^{2} / a_{N}^{2}\right),
$$

where the $m_{j}$ 's are nonnegative integers and $M=\left(m_{1}, \ldots, m_{N}\right)$ is a multi-index. The corresponding eigenfunctions are

$$
\phi_{M}(x)=\prod_{j=1}^{N} \cos \left(\pi m_{j} x / a_{j}\right) .
$$

For simple rectangles, every $l_{M}$ is a simple eigenvalue. We also write $d_{M}=$ $\left(h^{\prime}(\gamma)-1\right) / l_{M}$.

Let $S$ be the closure in $\mathbf{R}_{+} \times X$ of the set of all nonconstant solutions to (2.1)(2.2). Let $C_{M}$ be the connected component of $S$ containing the bifurcation point $\left(d_{M}, \gamma\right)$. In the neighborhood of $\left(d_{M}, \gamma\right), C_{M}$ is a smooth curve transversal to $\Gamma$. The continuum $C_{M}$ of the nonconstant solutions exists globally with respect to $d$ in the following sense.

THEOREM 4. Suppose that (A.1)-(A.5) are satisfied and let $\Omega=R$ be a simple rectangle. Then

$$
\operatorname{Proj}_{\mathbf{R}_{+}} C_{M} \supset\left(0, d_{M}\right]
$$

for each $M \neq(0, \ldots, 0)$. Here $\operatorname{Proj}_{\mathbf{R}_{+}}: \mathbf{R}_{+} \rightarrow X \times \mathbf{R}_{+}$is the projection operator.

From the proof, we observe a few facts.

First, let $\alpha, \beta$ be such that $0<\alpha \leq \beta$ and put $A=\{(d, u) \in S \cup \Gamma \mid \alpha \leq d \leq \beta\}$. Then $A$ is compact in $\mathbf{R}_{+} \times X$. Indeed, let $G$ be the Green operator of $I-\Delta$ subject to Neumann boundary conditions. Then $G$ is a compact operator on $X$ and solutions $(d, u)$ of $(2.1)-(2.2)$ are in one-to-one correspondence with solutions $(\lambda, w) \in(1,+\infty) \times X$ of

$$
w=\lambda G w+(\lambda-1)\left(h^{\prime}(\gamma)-1\right)^{-1} G[\eta(w)]
$$

through the relation $\lambda=1+\left(h^{\prime}(\gamma)-1\right) d^{-1}$ and $w=u-\gamma$. Since the right-hand side of (3.9) defines a compact operator on $(1,+\infty) \times X$, we have the claim.

Secondly, the a priori estimates given by Theorem 2 and the uniqueness by Theorem 3 remain valid for rectangles. This is because both (a) and (b) of Lemma 2.2 hold also for rectangles. In fact, by applying some standard reflecting arguments, we see that (a) is valid for Lipschitz domains [6], and (b) for rectangles is proved by making use of the Fourier series expansion and Marcinkiewicz' theorem on the Fourier multipliers [7].

Now we can verify Theorem 4 immediately by combining the next lemma with the above observations.

LEMMA 3.2. For simple rectangles, $C_{M}$ is not compact in $\mathbf{R}_{+} \times X$ for each $M \neq(0, \ldots, 0)$.

ProOF. We proceed by induction on $N$. For $N=1$, the assertion is well known (e.g., Rabinowitz [12, Theorem 2.3]). 
Assuming that the lemma is true for $N-1$, we verify the assertion for $N$ by adapting Nishiura's symmetry argument [11]. First note that if $C_{M}$ contains a bifurcation point $\left(d_{J}, \gamma\right), J=\left(j_{1}, \ldots, j_{N}\right)$, such that $j_{l}=0$ for some $l$, then $C_{M}$ is noncompact. Indeed, any solution to (2.1)-(2.2) in an $N-1$ dimensional rectangle $R_{N-1}$ can be naturally viewed as a solution in the rectangle $R_{N}$ of dimension $N$ such that $\partial R_{N} \supset R_{N-1}$, so that by the assumption of induction, the noncompactness follows.

Suppose now that $C_{M}$ is compact in $\mathbf{R}_{+} \times X$; then Rabinowitz' alternative [12, Theorem 1.3] implies that $C_{M}$ contains another bifurcation point $\left(d_{J}, \gamma\right)$. We pick up $M^{*}$ such that $\left|M^{*}\right|=\max \left\{|J| \mid\left(d_{J}, \gamma\right) \in C_{M}\right\}$, where $|J|=j_{1}+\cdots+j_{N}$. From the above observation we may assume $m_{1}^{*} \cdots m_{N}^{*} \neq 0$. Let us consider (2.1)-(2.2) in a smaller rectangle

$$
R^{\prime}=\left\{\left(x_{1}, \ldots, x_{N}\right) \mid 0<x_{j}<a_{j} / m_{j}^{*}, j=1, \ldots, N\right\} .
$$

Then $l_{I}^{\prime}=\pi^{2} \sum_{j=1}^{N}\left(a_{j} / m_{j}^{*}\right)^{-2}, I=(1, \ldots, 1)$, is a simple eigenvalue of $-\Delta$ in $R^{\prime}$ and $d_{I}^{\prime}=(h(\gamma)-1) / l_{I}^{\prime}\left(=d_{M}\right)$ gives a bifurcation point for the problem in $R^{\prime}$. Let $C_{I}^{\prime}$ be the component of $S^{\prime}$ containing $\left(d_{I}^{\prime}, \gamma\right)$, where $S^{\prime}$ is the counterpart of $S$. By reflection with respect to the sides of the rectangle, any solution in $R^{\prime}$ gives rise to a solution in $R$. Thus if $C_{I}^{\prime}$ is noncompact, then $C_{M}$ contains a noncompact subset; so assume $C_{I}^{\prime}$ is also compact. Then $C_{I}^{\prime}$ contains another bifurcation point $\left(d_{K}^{\prime}, \gamma\right), K=\left(k_{1}, \ldots, k_{N}\right)$, with $k_{1} k_{2} \cdots k_{N} \neq 0$ (otherwise, $C_{I}^{\prime}$ is noncompact as we have seen above). Since $I \neq K$, at least one of $k_{j}$ 's is greater than one. Now, $C_{M}$ contains the bifurcation point $\left(d_{K^{*}}, \gamma\right), K^{*}=\left(m_{1}^{*} k_{1}, \ldots, m_{N}^{*} k_{N}\right)$, which contradicts the maximality of $\left|M^{*}\right|$. Therefore the proof is completed. Q.E.D.

4. Activator-inhibitor systems. The objective of this section is to derive a priori estimates for solutions to activator-inhibitor systems specified below. This is done in subsection 4.1. By making use of the estimates we shall show in subsection 4.2 that assertions analogous to Theorems 3 and 4 hold also for such systems under certain conditions. Moreover, we prove that the inhibitor $v$ is close to a constant if its diffusion constant is sufficiently large.

Let $d, D$, and $\nu$ be positive constants. A system of equations in positive functions $u$ and $v$,

$$
\begin{aligned}
& \begin{array}{l}
d \Delta u-u+\Phi(u, v)=0 \\
D \Delta v-\nu v+\Psi(u, v)=0
\end{array} \\
& \frac{\partial u}{\partial n}=\frac{\partial v}{\partial n}=0 \quad \text { on } \partial \Omega,
\end{aligned} \quad \text { in } \Omega,
$$

is said to be an activator-inhibitor system if $\Phi$ and $\Psi$ satisfy the following conditions:

$$
\begin{gathered}
\Phi \in C^{1}([0,+\infty) \times(0,+\infty)), \\
\Phi(u, v) \geq 0, \quad \frac{\partial \Phi}{\partial u}(u, v)>0, \quad \frac{\partial \Phi}{\partial v}(u, v)<0 \quad \text { for } u \geq 0, v>0 ; \\
\Psi \in C^{1}([0,+\infty) \times(0,+\infty)), \\
\Psi(u, v) \geq 0, \quad \frac{\partial \Psi}{\partial u}(u, v)>0, \quad \frac{\partial \Psi}{\partial v}(u, v) \leq 0 \quad \text { for } u \geq 0, v>0 .
\end{gathered}
$$

We call $u$ an activator and $v$ an inhibitor. 
In what follows, we put further restrictions on $\Phi$ and $\Psi$. One is concerned with the growth rate in $u$ of $\Phi$ :

(B.3) There exist a nonincreasing function $A: \mathbf{R}_{+} \rightarrow \mathbf{R}_{+}$and $p>1$ such that for each $v>0, \Phi(u, v) \leq A(v) u^{p}$ if $u$ is sufficidently large.

Others are concerned with the relationship between $\Phi$ and $\Psi$ :

(B.4) There are $\alpha \geq 1, \beta \geq 1, \delta_{0}>0$, and $b_{0}>0$ such that $\Psi(u, v) \geq$ $b_{0} \Phi(u, v)^{\alpha} v^{\beta}$ if $u \geq \delta_{0}^{-1}$ or $0<v \leq \delta_{0}$.

Moreover, let $p$ satisfy

$$
\begin{array}{ll}
p \in(1, N /(N-2 \alpha)) & \text { if } 1 \leq \alpha<N / 2, \\
p \in(1,+\infty) & \text { if } \alpha \geq N / 2 .
\end{array}
$$

REMARK 4.1. (a) In view of $\Psi_{v} \leq 0$ and (B.3), for each $\delta>0$ we can find $B_{\delta}>0$ and $b_{1}$ such that

$$
\Phi(u, v) \leq B_{\delta} u^{p} v^{-\alpha / \beta}+b_{1}
$$

for all $u \geq 0$ and $v \geq \delta$.

(b) In the case of the Gierer-Meinhardt system (1.1)-(1.3), all assumptions (B.1)(B.5) are satisfied if (1.11) holds by choosing $\alpha=r / p$ and $\beta=q r / p-s$.

Now let us put

$$
\begin{aligned}
& f(u, v)=\Phi(u, v)-u \\
& g(u, v)=\Psi(u, v)-\nu v
\end{aligned}
$$

which is the "kinematic part" of (4.1)-(4.2). In virtue of (B.1), $f(u, v)=0$ defines a $C^{1}$ function $v=k(u)$ such that $f(u, k(u)) \equiv 0$. Similarly, (B.2) yields the existence of a function $v=l(u)$ of class $C^{1}$ satisfying $g(u, l(u))=0$ for all $u \geq 0$ and $l^{\prime}(u)>0$ for $u>0$.

4.1. A priori estimates. Throughout this subsection we shall always assume that solutions $(u, v)$ to $(4.1)-(4.3)$ are of class $C^{2}(\Omega) \cap C^{1}(\bar{\Omega})$. It is convenient to introduce the following notations:

$$
\begin{aligned}
& \left\{\begin{array}{l}
U_{*}=\min u(x), \quad U^{*}=\max u(x), \\
V_{*}=\min v(x), \quad V^{*}=\max v(x) ;
\end{array}\right. \\
& \inf _{u \geq 0, v>0} \Phi(u, v) \text { and } \quad \tau=\inf _{u \geq 0, v>0} \Psi(u, v) .
\end{aligned}
$$

Note that $l(v) \geq \tau / \nu$ since $v=l(u)$ solves $\nu v=\Psi(u, v)$.

We are going to bound $\|u\|_{C^{\theta}(\bar{\Omega})}$ and $\|v\|_{C^{\theta}(\bar{\Omega})}$ in terms of $d, D$, and $V_{*}$, (Proposition 4.3 and Theorem 6(a) below). As a corollary we shall give explicit a priori estimates which are valid for all positive solutions in the case of $\sigma+\tau>0$ and in the case of $\sigma=\tau=0$ under further restrictions on $\Phi$ and $\Psi$. Let us begin with the next important observation (the proof of (4.9) is similar to that of Proposition 1.1 in $[16] ;(4.10)$ is verified in the same way as in the proof of Lemma $1.2(1.13)$ in [17]).

Proposition 4.2. Any solution $(u, v)$ to (4.1)-(4.3) satisfies

$$
\begin{gathered}
u(x)>\sigma, \\
l\left(U_{*}\right) \leq v(x) \leq l\left(U^{*}\right)
\end{gathered}
$$


for all $x \in \bar{\Omega}$. In particular,

$$
V_{*}>l(\sigma) .
$$

To state our main results in this section, we define an integer $m$ as

$$
m=\left\{\begin{array}{l}
0 \text { if } \alpha>N / 2 ; \\
\min \left\{j \in Z \mid p^{j}>2 \alpha /[N-(N-2 \alpha) p]\right\} \quad \text { if } 1 \leq \alpha \leq N / 2 .
\end{array}\right.
$$

Note that $m \geq 1$ if $1 \leq \alpha \leq N / 2$ since $1<p<N /(N-2)$ by virtue of (B.5), where $N /(N-2 \alpha)$ stands for $+\infty$ if $2 \alpha=N$.

Recall that $\Lambda_{m}=\sum_{j=0}^{m} p^{j}$. Let $\Lambda_{-1}=0$.

With these preliminaries we can now state the main results:

THEOREM 5. Assume that (B.1)-(B.5) hold.

(a) Let $\sigma+\tau>0$. Then any solution to (4.1)-(4.3) satisfies

$$
\|u\|_{C^{\theta}(\bar{\Omega})} \leq C_{1} K(l(\sigma)) \max \left(1, d^{-\Lambda_{m}}\right)
$$

where

$$
K(t)=\max \left(1, t^{-\Lambda_{m-1} \beta / \alpha}\right) \max \left(1, t^{p^{m}(1-\beta) / \alpha}\right) .
$$

(b) Let $\alpha>N / 2$ and $\beta=1$. Then for any solution to (4.1)-(4.3) it holds that

$$
\|u\|_{C^{\theta}(\bar{\Omega})} \leq C_{1} \max \left(1, d^{-1}\right) .
$$

Here, $C_{1}>0$ and $\theta \in(0,1)$ do not depend on $(u, v), d$ or $D$.

Note that (4.15) is valid even for $\sigma=\tau=0$.

Observing that $l(\sigma)>0$ if $\sigma+\tau>0$ since $l(u)>l(0) \geq \tau / \nu$ for $u>0$, and hence $V_{*}$ is uniformly bounded away from 0 by $l(\sigma)>0$ in virtue of (4.11); and that if $m=0$ and $\beta=1$ then $K(t)=1$, we obtain Theorem 5 immediately from the following proposition.

Proposition 4.3. Under assumptions (B.1)-(B.5), any solution to (4.1)-(4.3) satisfies

$$
\|u\|_{C^{\theta}(\bar{\Omega})} \leq C_{1} K\left(V_{*}\right) \max \left(1, d^{-\Lambda_{m}}\right)
$$

with $\theta \in(0,1), C_{1}>0$ independent of $(u, v), d$, and $D$.

We remark that combining (4.10) with (4.13), (4.15) or (4.16) yields upper bounds on $V^{*}$ which are independent of $D$. As for a priori estimates for $\|v\|_{C^{\theta}(\bar{\Omega})}$, we have the following Theorem 6 . To treat the case $\sigma=\tau=0$ we have to impose a further assumption on $\Psi$ :

(B.6) There exists a $\lambda>N / 2$ and an increasing function $B: \mathbf{R}_{+} \rightarrow \mathbf{R}_{+}$ such that $\Psi(u, v) \leq B(u) v^{-1 /(\lambda-1)}$ for sufficiently small $v>0$.

THEOREM 6. Assume that (B.1)-(B.5) hold.

(a) For any solution to (4.1)-(4.3), it holds that

$$
\|v\|_{C^{\theta}(\bar{\Omega})} \leq C_{2} \Psi\left(U^{*}, V_{*}\right) \max \left(1, D^{-1}\right) ;
$$

in particular, if $\sigma+\tau>0$ then

$$
\|v\|_{C^{\theta}(\bar{\Omega})} \leq C_{2} \Psi\left(C_{1} K(l(\sigma)) \max \left(1, d^{-\Lambda_{m}}\right), l(\sigma)\right) \max \left(1, D^{-1}\right) .
$$


(b) Let $\alpha>N / 2$ and $\beta=1$. If, in addition to (B.1)-(B.5), (B.6) is satisfied, then

$$
\|v\|_{C^{\theta}(\bar{\Omega})} \leq C_{2}\left[B\left(C_{1} \max \left(1, d^{-1}\right)\right)\right]^{(\lambda-1) / \lambda} \max \left(1, D^{-1}\right)
$$

holds for any solution to (4.1)-(4.3).

Here $\theta \in(0,1)$ and $C_{2}>0$ are independent of $(u, v), d$, and $D$. 6.

The rest of this subsection is devoted to proving Proposition 4.3 and Theorem

PROOF OF PROPOSITION 4.3. We shall obtain the bounds on Hölder norm from those on $W^{2, t}(\Omega)$ norm of solutions, $t>N / 2$, as in the proof of Theorem 2. find

Step 1. First we divide both sides of (4.2) by $v^{\beta}$ and then integrate over $\Omega$ to

$$
\beta D \int_{\Omega} v^{-\beta-1}|\nabla v|^{2} d x+\int_{\Omega} \Psi(u, v) v^{-\beta} d x=\nu \int_{\Omega} v^{1-\beta} d x
$$

Since $\Phi(u, v) \leq \Phi\left(\delta_{0}^{-1}, \delta_{0}\right)$ if $u \leq \delta_{0}^{-1}$ and $v \geq \delta_{0}$, it follows from (B.4) and (4.20) that

$$
\|\Phi(u, v)\|_{L^{\alpha}(\Omega)} \leq C \max \left(1, V_{*}^{(1-\beta) / \alpha}\right),
$$

where, as usual, $C$ denotes various positive constants independent of $(u, v), d$, and $D$.

Next we multiply both sides of $(4.1)$ by $u^{\alpha-1}$ and then integate over $\Omega$. Integration by parts leads to

$$
(\alpha-1) d \int_{\Omega} u^{\alpha-2}|\nabla u|^{2} d x+\int_{\Omega} u^{\alpha} d x=\int_{\Omega} u^{\alpha-1} \Phi(u, v) d x .
$$

By Hölder's inequality, the right-hand side does not exceed $\|u\|_{L^{\alpha}(\Omega)}^{\alpha-1}\|\Phi(u, v)\|_{L^{\alpha}(\Omega)}$, and hence

$$
\|u\|_{L^{\alpha}(\Omega)} \leq\|\Phi(u, v)\|_{L^{\alpha}(\Omega)} .
$$

Now let us put

$$
g_{1}(x)=u(x)+d^{-1}[\Phi(u(x), v(x))-u(x)] .
$$

Since $\left|1-d^{-1}\right| \leq \max \left(1, d^{-1}\right),\left|g_{1}\right| \leq \max \left(1, d^{-1}\right) u+d^{-1} \Phi(u, v)$; therefore, from (4.21) and (4.23) we have

$$
\left\|g_{1}\right\|_{L^{\alpha}(\Omega)} \leq C \max \left(1, V_{*}^{(1-\beta) / \alpha}\right) \max \left(1, d^{-1}\right) .
$$

Note that (4.1) is written in the form $\Delta u-u+g_{1}=0$. We would like to apply Lemma 2.2. First consider the case $\alpha>1$. Then by (2.9) we see that

$$
\|u\|_{W^{2, \alpha}(\Omega)} \leq C\left\|g_{1}\right\|_{L^{\alpha}(\Omega)} .
$$

In particular, if $\alpha>N / 2$, then we get (4.16) with $m=0$ by virtue of (4.25) and (4.26) together with the Sobolev embedding theorem. Thus in what follows we assume $\alpha \leq N / 2$. In view of the Sobolev embedding theorem, we have from (4.26) that

$$
\|u\|_{L^{t}(\Omega)} \leq C\left\|g_{1}\right\|_{L^{\alpha}(\Omega)}
$$


where $1 / t=1 / \alpha-2 / N$ if $\alpha \in(1, N / 2)$, and $1<t<+\infty$ if $\alpha=N / 2$. Secondly, consider the case $\alpha=1$. Then by the same reasoning as in Step 1 of the proof of Proposition 2.1, we get (4.27) with $1<t<N /(N-2)$.

Consequently, for $\alpha \in[1, N / 2]$ we have (4.27), where $1 / t=1 / \alpha-2 / N$ if $\alpha \in$ $(1, N / 2)$, and $1 / t>1 / \alpha-2 / N$ if $\alpha=1$ or $N / 2$.

Step 2 . Now it is easily seen that the same bootstrapping argument as in Steps 2 and 3 of the proof of Proposition 2.1 does work for this case provided that $1<p<$ $N /(N-2 \alpha)$. Hence we omit the details and only point out that the counterpart of the crucial estimate $(2.16)$ can be derived as follows.

$$
\begin{aligned}
\left\|g_{1}\right\|_{L^{t / p}(\Omega)} & \leq \max \left(1, d^{-1}\right)\|u\|_{L^{t / p}(\Omega)}+d^{-1}\|\Phi(u, v)\|_{L^{t / p}(\Omega)} \\
& \leq \max \left(1, d^{-1}\right)\left(C\|u\|_{L^{t}(\Omega)}+B_{\delta} V_{*}^{-\beta / \alpha}\left\|u^{p}\right\|_{L^{t / p}(\Omega)}+C\right) \\
& \leq C \max \left(1, d^{-1}\right) \max \left(1, V_{*}^{-\beta / \alpha}\right)\left(\|u\|_{L^{t}(\Omega)}^{p}+C\right),
\end{aligned}
$$

where we have used (4.4). In view of (4.25) and (4.27), we thus have an estimate for $\left\|g_{1}\right\|_{L^{t / p}(\Omega)}$ in terms of $\left\|g_{1}\right\|_{L^{\alpha}(\Omega)}$, so in terms of $V_{*}$ and $d$. Q.E.D.

PROOF OF THEOREM 6. First of all, we observe that by the same reasoning as in proving (4.23)

$$
\nu\|v\|_{L^{t}(\Omega)} \leq\|\Psi(u, v)\|_{L^{t}(\Omega)}
$$

for $t \geq 1$. Hence, putting

$$
g_{2}(x)=v(x)+D^{-1}(\Psi(u(x), v(x))-\nu v(x)),
$$

we find that

$$
\left\|g_{2}\right\|_{L^{t}(\Omega)} \leq C \max \left(1, D^{-1}\right)\|\Psi(u, v)\|_{L^{t}(\Omega)}
$$

Since (4.2) is written in the form $\Delta v-v+g_{2}=0$, we are able to apply Lemma 2.2 and the Sobolev embedding theorem to see that if $t>N / 2$, then

$$
\|v\|_{C^{\theta}(\bar{\Omega})} \leq C\|v\|_{W^{2, t}(\Omega)} \leq C\left\|g_{2}\right\|_{L^{t}(\Omega)} .
$$

In view of (B.2), we have $\Psi(u(x), v(x)) \leq \Psi\left(U^{*}, V_{*}\right)$ for all $x \in \bar{\Omega}$, so that $\|\Psi(u, v)\|_{L^{t}(\Omega)} \leq|\Omega|^{1 / t} \Psi\left(U^{*}, V_{*}\right)$. Therefore (a) is proved by (4.29) and (4.30) together with this observation.

For the verification of (b), it is sufficient to show that

$$
\|\Psi(u, v)\|_{L^{\lambda}(\Omega)} \leq\left[\nu|\Omega|\left(B\left(U^{*}\right)+C\right)^{\lambda-1}\right]^{1 / \lambda}
$$

since $\lambda>N / 2$. To do this, we first observe that

$$
\int_{\Omega} \Psi(u, v) v^{-1} d x \leq \nu|\Omega|
$$

by putting $\beta=1$ in (4.20). Therefore, writing $\Psi(u, v)^{\lambda}=\left(\Psi(u, v)^{\lambda-1} v\right)\left(\Psi(u, v) v^{-1}\right)$, we have (4.31) from (4.32) because

$$
\Psi(u, v)^{\lambda-1} v=\left(\Psi(u, v) v^{1 /(\lambda-1)}\right)^{\lambda-1} \leq(B(u)+C)^{\lambda-1}
$$

by virtue of (B.6). Q.E.D.

REMARK 4.4. In the case of the Gierer-Meinhardt system, (B.6) is satisfied if $0 \leq s<2 /(N-2)$ by choosing $\lambda>N / 2$ such that $s \leq(\lambda-1)^{-1}$. Therefore, we have Theorem 1 readily from Theorems 5 and 6 in view of Remark 4.1(b).

4.2. Some applications of a priori estimates. First, we show that if the activator diffuses very fast, then the only possible steady state is spatially homogeneous. 
THEOREM 7. In addition to (B.1)-(B.5), assume that $\sigma+\tau>0$. Then there is $d^{*}>0$, independent of $D$, such that (4.1)-(4.3) has no nonconstant positive solution if $d>d^{*}$.

In the case of $\sigma=\tau=0$, let $\alpha>N / 2$ and $\beta=1$. Then for each $\delta>0$ small, there exists $d^{*}>0$, depending on $\delta$ but not on $D$, such that if $d>d^{*}$ then (4.1)-(4.3) has no nonconstant solution satisfying $V_{*}=\min v>\delta$.

This theorem can be verified in a way analogous to that in the proof of Theorem 3 , or more precisely, by the same reasoning as in $\S 3$ of [16]; so we do not enter into details but only remark that the derivatives $\Phi_{u}, \Phi_{v}, \Psi_{u}$, and $\Psi_{v}$ remain bounded in absolute value as long as $(u, v)$ stays in bounded subsets of $\mathbf{R}_{+} \times \mathbf{R}_{+}$and $v \geq \delta>0$ and that $\max u(x)$ and $\max v(x)$ are estimated by constants independent of $D$.

Next, we consider the existence of nonconstant solutions by applying bifurcation theory under the following assumption:

(B.7) The equation $f(u, v)=g(u, v)=0$ has exactly one positive solution $(u, v)=(\bar{u}, \bar{v})$.

This is equivalent to saying that $u=\Phi(u, l(u))$ has a unique positive solution $u=\bar{u}$, since $v=l(u)$ solves $g(u, v)=0$. Thus, (B.7) is fulfilled if, for instance,

$$
\Psi(u, v)=(\Phi(u, v)-\sigma)^{\alpha} v^{\beta} .
$$

Indeed, substituting $v=l(u)$ into (4.33) gives $\Phi(u, l(u))=\left(\nu l(u)^{1-\beta}\right)^{1 / \alpha}+\sigma ;$ since the right-hand side is nonincreasing, $u=\Phi(u, l(u))$ has a unique positive solution. Note also that the Gierer-Meinhardt system (1.1)-(1.3) satisfies (B.7) under the general condition (1.4).

Now let $\Omega$ be a simple rectangle as in Theorem 4. We fix $D>0$ and look for a pair $(d,(u, v)) \in \mathbf{R}_{+} \times X \times X$ satisfying (4.1)-(4.3), where $X$ is defined by (3.5). We note that $\Gamma=\{(d,(\bar{u}, \bar{v})) \mid d>0\}$ forms the branch of constant solutions.

For each positive eigenvalue $l_{M}$ of $-\Delta$, put

$$
d^{(M)}=l_{M}^{-1}\left[a_{11}+a_{12} a_{21} /\left(D l_{M}-a_{22}\right)\right],
$$

where $a_{11}=f_{u}(\bar{u}, \bar{v}), a_{12}=f_{v}(\bar{u}, \bar{v}), a_{21}=g_{u}(\bar{u}, \bar{v})$, and $a_{22}=g_{v}(\bar{u}, \bar{v})$. Observe that $a_{12}<0, a_{21}>0$, and $a_{22}<0$ by (B.1) and (B.2). Then the linear part

$$
\mathcal{L}(d)=\left(\begin{array}{cc}
d \Delta+a_{11} & a_{12} \\
a_{21} & D \Delta+a_{22}
\end{array}\right)
$$

of the operation in (4.1)-(4.3) around the constant solution $(\bar{u}, \bar{v})$ has 0 as an eigenvalue if and only if $a_{11}>0$ and $d=d^{(M)}$ with

$$
l_{M}>\left(a_{11} a_{22}-a_{12} a_{21}\right) /\left(a_{11} D\right) .
$$

Moreover, for given $d^{(M)}>0$, there exists at most one $J$ such that $d^{(J)}=d^{(M)}$ and $J \neq M$. It is easy to see that (a) if $d^{(M)} \neq d^{(J)}$ for all $J \neq M$, then 0 is a simple eigenvalue of $\mathcal{L}\left(d^{(M)}\right)$; and (b) otherwise, $\mathcal{L}\left(d^{(M)}\right)$ has 0 as an eigenvalue of algebraic multiplicity 2 . We call $d^{(M)}$ simple if (a) occurs, otherwise it is called double. Let $d^{(M)}>0$ be simple. Then $\mathcal{L}\left(d^{(M)}\right)$ satisfies the nondegeneracy condition of Crandall and Rabinowitz [4, Theorem 2.4] as $d$ changes through $d^{(M)}$; consequently, $\left(d^{(M)},(\bar{u}, \bar{v})\right)$ is a bifurcation point: there exists a one-parameter family 
of nonconstant solutions $\left(d_{M}(\varepsilon),\left(u_{M}(\varepsilon), v_{M}(\varepsilon)\right)\right)$ such that $d_{M}(\varepsilon)=d^{(M)}+O(\varepsilon)$, $u_{M}(\varepsilon)=\bar{u}+\varepsilon \phi_{M}(x)+o(\varepsilon)$, and $v_{M}(\varepsilon)=\bar{v}+\varepsilon b_{M} \phi_{M}(x)+o(\varepsilon)$ with $|\varepsilon|<\varepsilon_{0}$ and $b_{M}=a_{12} /\left(D l_{M}-a_{22}\right)$.

Let $S$ be the closure in $\mathbf{R}_{+} \times X \times X$ of the set of nonconstant solutions to (4.1)(4.3), and $C_{M}$ be the connected component of $S$ containing $\left(d^{(M)},(\bar{u}, \bar{v})\right)$. Then we can verify the following theorem in the same way as in the proof of Theorem 4 since the reasoning in proving Lemma 3.2 works for systems of equations such as (4.1)-(4.3) (see also [11 or 16] where the case $N=1$ is proved).

THEOREM 8. Let $\Omega$ be a simple rectangle. Suppose that (B.7) is satisfied in addition to (B.1)-(B.5). Let $\sigma+\tau>0$ and $a_{11}=\Phi_{u}(\bar{u}, \bar{v})-1>0$. Then for each simple $d^{(M)}$ it holds that

$$
\operatorname{Proj}_{\mathbf{R}_{+}} C_{M} \supset\left(0, d^{(M)}\right] .
$$

Finally, we would like to show that if $D$ is sufficiently large, then $v$ is close to a constant. Let us decompose $v$ as $v(x)=v_{0}+\psi(x)$, where $v_{0}=|\Omega|^{-1} \int_{\Omega} v d x$ and hence $\int_{\Omega} \psi d x=0$. Then we can bound $\max |\psi(x)|$ as follows.

THEOREM 9. Under assumptions (B.1)-(B.5), for each $\delta>0$ there exists a constant $C>0$ independent of $D$ such that for any solution to (4.1)-(4.3) satisfying $\min v \geq \delta$ the following estimate holds:

$$
\max |\psi(x)| \leq C / D .
$$

Moreover, $C$ is independent of $\delta$ if $\sigma+\tau>0$ or if $\alpha>N / 2, \beta=1$, and (B.6) is satisfied.

ProOF. Let us begin with recalling the known result on the boundedness of weak solutions to linear elliptic equations (e.g., Théorème 1 of Stampacchia [15]).

Let $t>N / 2$ and $h_{0} \in L^{t}(\Omega)$ with $\int_{\Omega} h_{0} d x=0$. If $w \in W^{1,2}(\Omega)$ is a weak solution to

$$
\Delta w+h_{0}=0 \quad \text { in } \Omega, \quad \frac{\partial w}{\partial n}=0 \quad \text { on } \partial \Omega,
$$

then, for some $C>0$ depending only on $\Omega$ and $t$,

$$
\|w\|_{L^{\infty}(\Omega)} \leq C\left(\left\|h_{0}\right\|_{L^{t}(\Omega)}+\|w\|_{L^{2}(\Omega)}\right) .
$$

Now putting $g_{0}(x)=\Psi(u(x), v(x))-\nu v(x)$, we note that $w=\psi$ satisfies (4.37) with $h_{0}=D^{-1} g_{0}$, and hence (4.38) reads as

$$
\|\psi\|_{L^{\infty}(\Omega)} \leq C\left(D^{-1}\left\|g_{0}\right\|_{L^{t}(\Omega)}+\|\psi\|_{L^{2}(\Omega)}\right) .
$$

We claim that (a) $\|\psi\|_{L^{2}(\Omega)} \leq C D^{-1}\left\|g_{0}\right\|_{L^{t}(\Omega)}$ and (b) $\left\|g_{0}\right\|_{L^{t}(\Omega)} \leq C_{\delta}$ if $V_{*}>\delta>$ 0 , where $C$ and $C_{\delta}$ do not depend on $D$. Combining (4.39) with (a) and (b) yields (4.36).

To prove (a), we multiply both sides of $D \Delta \psi+g_{0}=0$ by $\psi$ and then integrate over $\Omega$. Integration by parts and Hölder's inequality give

$$
D \int_{\Omega}|\nabla \psi|^{2} d x=\int_{\Omega} g_{0} \psi d x \leq\left\|g_{0}\right\|_{L^{t}(\Omega)}\|\psi\|_{L^{t^{\prime}}(\Omega)},
$$


where $t^{\prime}=t /(t-1)$. Putting $2^{*}=2 N /(N-2)$ if $N \geq 3$ and $2^{*}=t^{\prime}$ if $N=2$, we see that $2^{*} \geq t^{\prime}$ and hence, with the aid of Hölder's inequality and the Sobolev embedding theorem, that

$$
\|\psi\|_{L^{t^{\prime}}(\Omega)} \leq C\|\psi\|_{L^{2^{*}}(\Omega)} \leq C\|\psi\|_{W^{1,2}(\Omega)}
$$

Since $\int_{\Omega} \psi d x=0$, it holds that

$$
\begin{aligned}
\|\psi\|_{W^{1,2}(\Omega)}^{2} & =\int_{\Omega} \psi^{2} d x+\int_{\Omega}|\nabla \psi|^{2} d x \\
& \leq\left(1+c_{0}\right) \int_{\Omega}|\nabla \psi|^{2} d x
\end{aligned}
$$

by the Poincaré inequality (3.4). Therefore $\|\psi\|_{L^{t^{\prime}}(\Omega)} \leq C\|\nabla \psi\|_{L^{2}(\Omega)}$, and we have from (4.40) that $D\|\nabla \psi\|_{L^{2}(\Omega)} \leq C\left\|g_{0}\right\|_{L^{t}(\Omega)}$. Applying the Poincaré inequality again, we obtain (a).

Next we turn to the proof of (b). Since $0 \leq \Psi(u(x), v(x)) \leq \Psi\left(U^{*}, V_{*}\right)$ by (B.2), we see that $\|\Psi(u, v)\|_{L^{\infty}(\Omega)}$ is bounded uniformly in $D$ provided $V_{*}>\delta$ because of (4.16). In view of (4.28) we therefore have (b). If $\sigma+\tau>0$, then $V_{*}>l(\sigma)>0$ for all solutions, so $\left\|g_{0}\right\|_{L^{t}(\Omega)}$ is bounded by a constant independent of $\delta$; if $\alpha>N / 2$, $\beta=1$, and (B.6) holds, then $\left\|g_{0}\right\|_{L^{\lambda}(\Omega)}, \lambda>N / 2$, is bounded uniformly in $V_{*}$ by virtue of (4.31), (4.15), and (4.28). Thus the proof is complete. Q.E.D.

\section{REFERENCES}

1. S. Agmon, A. Douglis and L. Nirenberg, Estimates near the boundary for solutions of elliptic partial differential equations satisfying general boundary conditions. I, Comm. Pure Appl. Math. 12 (1959), 623-727.

2. R. Böhme, Die Lösung der Verzweigungsgleichungen für nichtlineare Eigenwertprobleme, Math. Z. 127 (1972), 105-126.

3. H. Brezis and W. A. Strauss, Semi-linear second-order elliptic equations in $L^{1}$, J. Math. Soc. Japan 25 (1973), 565-590.

4. M. G. Crandall and P. H. Rabinowitz, Bifurcation from simple eigenvalues, J. Funct. Anal. 8 (1971), 321-340.

5. A. Gierer and H. Meinhardt, A theory of biological pattern formation, Kybernetik 12 (1972), 30-39.

6. C. E. Kenig, Private communications.

7. J. Marcinkiewicz, Sur les multiplicateurs des séries de Fourier, Studia Math. 8 (1939), 78-91.

8. H. Meinhardt, Models of biological pattern formation, Academic Press, London and New York, 1982.

9. C. B. Morrey, Jr., Multiple integrals in the calculus of variations, Springer-Verlag, Berlin, Heidelberg and New York, 1966.

10. W.-M. $\mathrm{Ni}$, On the positive radial solutions of some semilinear elliptic equations on $\mathbf{R}^{n}$, Appl. Math. Optim. 9 (1983), 373-380.

11. Y. Nishiura, Global structure of bifurcating solutions of some reaction-diffusion systems, SIAM J. Math. Anal. 13 (1982), 555-593.

12. P. H. Rabinowitz, Some global results for nonlinear eigenvalue problems, J. Funct. Anal. 7 (1971), 487-513.

13. __ A bifurcation theorem for potential operators, J. Funct. Anal. 25 (1977), 412-424.

14. F. Rothe, Global solutions of reaction-diffusion systems, Lecture Notes in Math., vol. 1072, Springer-Verlag, Berlin, Heidelberg, New York and Tokyo, 1984. 
15. G. Stampacchia, Équations elliptiques à données discontinues, Séminaire Schwartz, 1960/61, no. 4.

16. I. Takagi, A priori estimates for stationary solutions of an activator-inhibitor model due to Gierer and Meinhardt, Tôhoku Math. J. 34 (1982), 113-132.

17. , Point-condensation for a reaction-diffusion system, J. Differential Equations 61 (1986), 208-249.

School of Mathematics, University of Minnesota, Minneapolis, MinNesota 55455

Mathematical Institute, Tôhoku University, SENDAi 980, JAPAN 